\title{
Dispersion and Symmetry of Bound States in the Shastry-Sutherland Model
}

\author{
Christian Knetter, Alexander Bühler, Erwin Müller-Hartmann, and Götz S. Uhrig \\ Institut für Theoretische Physik, Universität zu Köln, D-50937 Köln, Germany
}

(October 30, 2018)

\begin{abstract}
Bound states made from two triplet excitations on the Shastry-Sutherland (ShaSu) lattice are investigated. Based on the perturbative unitary transformation by flow equations quantitative properties like dispersions and qualitative properties like symmetries are determined. The high order results (up to $\left.\left(J_{2} / J_{1}\right)^{14}\right)$ permit to fix the parameters of $\mathrm{SrCu}_{2}\left(\mathrm{BO}_{3}\right)_{2}$ precisely: $J_{1}=6.16(10) \mathrm{meV}$, $x:=J_{2} / J_{1}=0.603(3), J_{\perp}=1.3(2) \mathrm{meV}$. At the border of the magnetic Brillouin zone (MBZ) a general double degeneracy is derived. An unexpected instability in the triplet channel at $x=0.63$ indicates a first order transition towards a triplet condensate, related to classical helical order.
\end{abstract}

PACS numbers: 75.40.Gb, 75.30.Kz, 75.50.Ee, 75.10.Jm

Dedicated to Prof. F. Wegner on occasion of his $60^{\text {th }}$ birthday.

Quantum antiferromagnets are at the center of research not only because of the high $T_{c}$ superconductors . Of particular interest are systems which do not have an ordered, Néel-type ground state. Their ground state is a spin liquid without long range spin order. Spin liquids are favored by low spin $\left(S=\frac{1}{2}\right.$ mostly), low coordination number $(Z \in\{2,3,4\} \Rightarrow D \in\{1,2\})$, and strong geometric frustration.

Dimer solids are transparent cases of spin liquids. In $D=1$, the generic example is the Majumdar-Ghosh model [1] of which Shastry and Sutherland found a $D=2$ generalization (ShaSu model) [2]. In both cases frustration is essential. Each spin is coupled to pairs of spins (dimers). If these pairs form singlets the couplings between dimers is without effect and the singlet-on-dimers product state is always an eigen state and for certain parameters the ground state [3,4, 2,5]. The systems are gapped. The elementary excited states are dressed $S=\frac{1}{2}$ $(D=1)$ [ or $S=1(D=2)$ entities. They interact strongly and form bound and antibound states in various spin channels. Due to its recent realization in

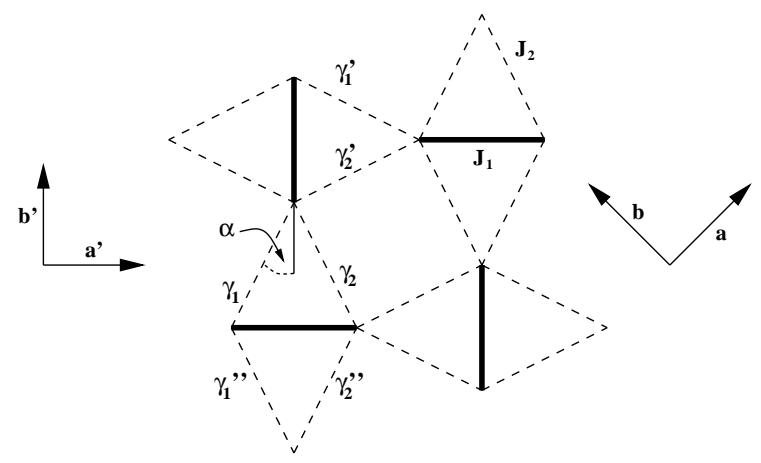

FIG. 1. Unit cell of the Shastry-Sutherland (ShaSu) lattice as realized in $\mathrm{SrCu}_{2}\left(\mathrm{BO}_{3}\right)_{2},(\mathbf{a}, \mathbf{b})$ are unit vectors; (a', $\mathbf{b}^{\prime}$ ) and the coefficients $\gamma$ are used to analyse Raman scattering.

$\mathrm{SrCu}_{2}\left(\mathrm{BO}_{3}\right)_{2}$ [6, 7 the $\mathrm{ShaSu}$ model (Fig. 1) is presently attracting enormous interest. The Hamiltonian reads

$$
H\left(J_{1}, J_{2}\right)=J_{1} \sum_{\langle\mathbf{i}, \mathbf{j}\rangle \text { dimer }} \mathbf{S}_{\mathbf{i}} \mathbf{S}_{\mathbf{j}}+J_{2} \sum_{\langle\mathbf{i}, \mathbf{j}\rangle \text { square }} \mathbf{S}_{\mathbf{i}} \mathbf{S}_{\mathbf{j}} .
$$

In this Letter we start from the dimer phase [5]. We focus on bound states formed from pairs of the elementary triplets and their symmetries, degeneracies, and dispersion. The perturbative unitary transformation \& based on flow equations [9] enables us to link smoothly and uniquely $H\left(J_{1}, J_{2}\right)$ at $x:=J_{2} / J_{1} \neq 0$ to an effective $H_{\text {eff }}$ conserving the number of triplets on dimers $\left[H_{\text {eff }}, H\left(J_{1}, 0\right)\right]=0$. This permits a clear distinction between the ground state (without triplets), the 1-triplet sector, the 2-triplet sector etc..

In terms of $H_{\text {eff }}$ the dynamics of one triplet is hopping $t_{h ; \mathbf{i}}\left(t_{v ; \mathbf{i}}\right)$ starting from a horizontal (vertical) dimer by $i_{x}$ dimers right and $i_{y}$ dimers up. Nothing else is possible due to triplet number conservation. The elements $t$ are computed in order 15 [5, 11.

The dynamics of two triplets at large distances is governed by 1-triplet hopping. At smaller distances a 2particle interaction occurs additionally given by $W_{h ; \mathbf{d} ; \mathbf{i}, \mathbf{d}^{\prime}}$ $\left(W_{v ; \mathbf{d} ; \mathbf{i}, \mathbf{d}^{\prime}}\right)$ starting with one triplet on a horizontal (vertical) dimer and another at distance $\mathbf{d}$. The action of $H_{\text {eff }}$ is to shift the triplets to $\mathbf{i}$ and to $\mathbf{i}+\mathbf{d}^{\prime}$. Nothing else is possible due to triplet number conservation. Since the total spin is conserved $(S \in\{0,1,2\})$ the distances are restricted to $\mathbf{d}, \mathbf{d}^{\prime}>\mathbf{0}$, i.e. $d_{x}>0$ or $d_{x}=0 \wedge d_{y}>0$, because the exchange parity is fixed.

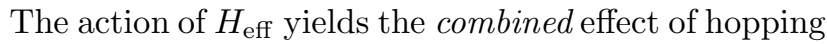
and interaction denoted by $A_{\mathbf{d} ; \mathbf{i}, \mathbf{d}^{\prime}}$. The true 2-triplet interaction is easily found by subtracting the 1-triplet hopping [5, 10, 11

$$
\begin{aligned}
W_{\mathbf{d} ; \mathbf{0}, \mathbf{d}^{\prime}} & =A_{\mathbf{d} ; \mathbf{0}, \mathbf{d}^{\prime}}-t_{\mathbf{d}^{\prime}-\mathbf{d}}-\delta_{\mathbf{d}^{\prime}, \mathbf{d}} t_{\mathbf{0}} \\
W_{\mathbf{d} ; \mathbf{d}-\mathbf{d}^{\prime}, \mathbf{d}^{\prime}} & =A_{\mathbf{d} ; \mathbf{d}-\mathbf{d}^{\prime}, \mathbf{d}^{\prime}}-t_{\mathbf{d}-\mathbf{d}^{\prime}}-\delta_{\mathbf{d}^{\prime}, \mathbf{d}} t_{\mathbf{0}} \\
W_{\mathbf{d} ;-\mathbf{d}^{\prime}, \mathbf{d}^{\prime}} & =A_{\mathbf{d} ;-\mathbf{d}^{\prime}, \mathbf{d}^{\prime}}-t_{-\mathbf{d}-\mathbf{d}^{\prime}} \\
W_{\mathbf{d} ; \mathbf{d}, \mathbf{d}^{\prime}} & =A_{\mathbf{d} ; \mathbf{d}, \mathbf{d}^{\prime}}-t_{\mathbf{d}+\mathbf{d}^{\prime}}
\end{aligned}
$$

(distinction $h / v$ omitted for clarity). Otherwise $A$ and $W$ are identical. The coefficients $W$ for $S \in\{0,1,2\}$ yield 
the complete 2-particle dynamics. We compute $W$ up to $x^{12}$, the coefficients for the lowest-lying states even up to $x^{14}$.

During the virtual processes [12] the triplet number is changed. Due to the frustration of the ShaSu lattice $H$ in (11) changes the number of triplets on the dimers at most by one [7, 10,11]. An excitation or a de-excitation on a horizontal (vertical) dimer is possible iff at least one of the vertical (horizontal) dimers on the left and right (above and below) are excited. This restriction implies that one triplet hops only in $x^{6}$ (cf. Figs. in [13, 11]).

Motion of two triplets together is much less restricted (cf. Fig. 2). Matrix elements occur in $x^{2}$ as first observed for total spin $S=2[14]$. But the dispersion of bound states starts only in $x^{3}$ (contrary to $x^{4}$ claimed in Ref. 15]). Two adjacent triplets interact linearly in $x$ $(-x$ for $S=0,-x / 2$ for $S=1, x / 2$ for $S=2)$. The energy of the initial and final state in each row in Fig. 2 differ by $\mathcal{O}(x)$. Hence both rows must be combined making it an $\left(x^{2}\right)^{2} / x=x^{3}$ process eventually. This applies to the 8 (anti)bound states derived from two triplets on (next) nearest neighbor dimers. The dispersion of any other state sets in at higher order.

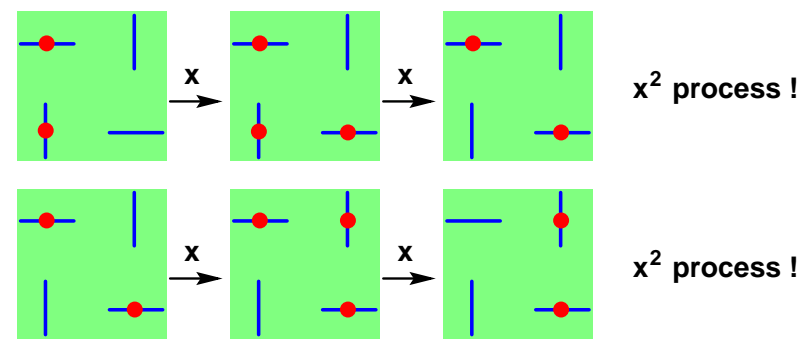

FIG. 2. Leading processes of correlated 2-triplet hopping. Dark dots are triplets, bars are dimers.

We use the following basis for the 2-triplet states

$$
|\mathbf{k}, \mathbf{d}, \sigma\rangle:=N^{-1 / 2} \sum_{\mathbf{r}} e^{(i(\mathbf{k}+\sigma \mathbf{Q})(\mathbf{r}+\mathbf{d} / 2))}|\mathbf{r}, \mathbf{r}+\mathbf{d}\rangle
$$

where $\mathbf{k}$ is the conserved total momentum in the magnetic Brillouin zone (MBZ) applying due to the two sublattices; $\sigma \in\{0,1\}, \mathbf{Q}:=(\pi, \pi), N$ is the number of dimers, $|\mathbf{r}, \mathbf{r}+\mathbf{d}\rangle$ denotes the state with triplets at $\mathbf{r}$ and at $\mathbf{r}+\mathbf{d}$. The distance $\mathbf{d}$ is restricted $\mathbf{d}>\mathbf{0}$, i.e. $d_{x}>0$ or $d_{x}=$ $0 \wedge d_{y}>0$. The matrix elements of $H_{\text {eff }}$ in the basis (3) are real due to translation and inversion symmetry.

Before the quantitative analysis of the bound states a qualitative aspect, a general double degeneracy at the border of the MBZ, shall be derived. To see this consider the combination of a shift by the dimer-dimer spacing along $\mathbf{a}^{\prime}(\mathrm{S})$, a reflection about $\mathbf{a}(\mathrm{R})$, and the inversion $\mathbf{r} \rightarrow-\mathbf{r}$ (I) (cf. Fig. 1). The combinations SR and I are symmetries of the Hamiltonian. For $k_{x}+k_{y}=\pi$ (part of the MBZ border) definition (3) implies for the total combination SRI the mapping

$$
|\mathbf{k}, \mathbf{d}, \sigma\rangle \rightarrow e^{i k_{x}+i \pi\left(d_{x}+d_{y}+\sigma+P S\right)}\left|\mathbf{k},-\left(\begin{array}{c}
d_{y} \\
d_{x}
\end{array}\right), 1-\sigma\right\rangle
$$

where $P \in\{0,1\}$ being unity iff $-\left(d_{y}, d_{x}\right)<\mathbf{0}$ so that the triplets must be swapped to pass from $-\left(d_{y}, d_{x}\right)$ to $\left(d_{y}, d_{x}\right)$. It is crucial that SRI links $\left|\mathbf{k},\left(d_{x}, d_{y}\right), 0\right\rangle$ and $\left|\mathbf{k},-\left(d_{y}, d_{x}\right), 1\right\rangle$ like a $2 \mathrm{D}$ rotation $\left(\begin{array}{cc}0 & -1 \\ 1 & 0\end{array}\right)$ up to a prefactor. Hence its eigen vectors are complex with linearly independent real and imaginary part and so are the simultaneous eigen vectors of SRI and $H_{\text {eff }}$. Because $H_{\text {eff }}$ is real the real and the imaginary part constitute in fact linearly independent eigen vectors to the same eigen value. The same double degeneracy is concluded for the other parts of the MBZ border by $\mathrm{S}$ and $90^{\circ}$ rotation (D). It is also valid in the 1 -triplet sector 11.

The double degeneracy at the MBZ border is interesting for analysing experiment, too. Degeneracy reduces the large number of energetically close states helping to resolve different bound states.

Since 1-triplet hopping is of higher order than interaction an analytic expansion for the energies of the bound states is possible. At finite order in $x$ only configurations contribute where the two triplets are not too far away from each other. Of course, higher orders imply larger, but still finite distances. In particular, the energies of the four states which evolve from neighboring triplets can be computed very well since their interaction is linear. Investigating the matrix elements shows that it is sufficient to study the distances $\mathbf{d} \in\{(0,1),(1,0),(1, \pm 1)\}$ for order 5 . To $x^{14}$ only $\mathbf{d} \in\{(1, \pm 2),(2, \pm 1),(0,2),(2,0),(2, \pm 2)\}$ must be added. So, for given total momentum only a finite $8 \times 8$ or $24 \times 24$ matrix has to be analysed. For illustration consider the elements $A_{(0,1) ; \mathbf{i},(2,1)}$ (the Fourier transform of $\mathbf{i}$ yields the momentum dependence.) connecting $(0,1)$ and $(2,1)$ which is $\mathcal{O}\left(x^{4}\right)$. By second order perturbation one sees that the resulting energy shift is $\left(x^{4}\right)^{2} / x=x^{7}$ only.

Furthermore, the elements connecting shorter distances to longer distances and the elements among longer distances do not need to be known to very high order. Consider again the process $(0,1) \leftrightarrow(2,1)$. In order $x^{7}$ the element $A_{(0,1) ; \mathbf{i},(2,1)}$ must be known only in $x^{4}$ and $A_{(2,1) ; \mathbf{i},(2,1)}$ only in $x^{1} ;$ in order $x^{9}$ the element $A_{(0,1) ; \mathbf{i},(2,1)}$ must be known only up to $x^{6}$ and $A_{(2,1) ; \mathbf{i},(2,1)}$ only in $x^{3}$ and so on.

We have analysed the dispersions in $x^{5}$ of the four states bound linearly in $x$ in the MBZ. Fukumoto's results are mostly confirmed [16,17]. At particular points of high point group symmetry $((0,0),(0, \pi),(\pi / 2, \pi / 2))$ the Hamiltonian splits into six blocks corresponding to different representations of the square point group $4 \mathrm{~mm}$. At these points the analysis up to $x^{14}$ is carried out [17]. The symmetries are classified according to the irreducible representations (four $1 \mathrm{D}$, one $2 \mathrm{D}$ ) of the point group 
$4 \mathrm{~mm} \Gamma_{1}(1), \Gamma_{2}\left(x^{2}-y^{2}\right), \Gamma_{3}(x y), \Gamma_{4}\left(x y\left(x^{2}-y^{2}\right)\right), \Gamma_{5}(x, y)$ where simple polynomials are given in brackets to show the transformation behavior.

The extrapolated energies are depicted in Figs. 3 ( $S=$ $0)$ and $1(S=1)$ as functions of $x$. For those energies which stay separated from the 2-particle continuum Dlog-Padé approximants are used successfully 18]. The results are stable under changes of the polynomial degrees. The energies close to the continuum (here simply twice the gap $\Delta$ between the ground state and the elementary triplet at $\mathbf{k}=\mathbf{0}$ ) are given with less reliability by the truncated series or by a non-defective Dlog-Padé approximant.

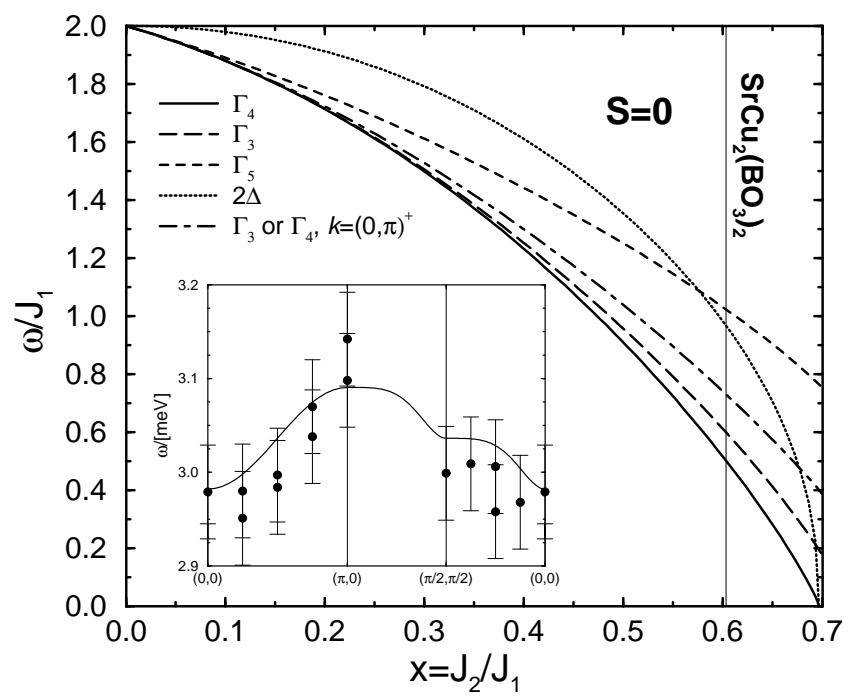

FIG. 3. Energy of the lowest-lying $S=0$ states. Curves refer to $\mathbf{k}=\mathbf{0}$ except the dashed-dotted one. The dotted curve displays the continuum at $2 \Delta$. Inset: 1 -triplet dispersion. Theory at $x=0.603, J_{1}=6.16 \mathrm{meV}$; data from Ref. [19], experimental errors at least as large as indicated.

In Figs. 3, 1 the modes are sorted in energetically ascending order for small values of $x$ : solid, long dashed, and short dashed curves. The $\Gamma_{5}$ modes are naturally degenerate. The double degeneracy for $\mathbf{k}=(0, \pi)$ does not result from the point group but originates from the complex conjugation as explained above. The dasheddotted curve at $(0, \pi)$ has to be compared to the solid and the long-dashed curve to assess the dispersion of these two modes from $\mathbf{0}$ to $(0, \pi)$. While for $S=0$ this dispersion always has the expected behavior with $\omega(\mathbf{0})<\omega((0, \pi))$ the energies for $S=1$ are reversed for small values of $x$ (cf. [16]). Only above $x \approx 0.55$ the relation $\omega(\mathbf{0})<\omega((0, \pi))$ holds for $S=1$.

We do not agree with Ref. [16] that the two lowest states are of $s$-wave type since this would imply that they are $\Gamma_{1}$. Instead the $S=0,1$ states are odd under reflection about a' (R1) or about b' (R2) (cf. Fig. 1). For $S=0$, the lowest state is even under SD and the second



FIG. 4. As in Fig. 3 for $S=1$. Inset: Magnetic susceptibility. Theory (dashed) with directional $\mathrm{rms}$ average $g=2.13$ [20], $x, J_{1}$ as in Fig. 3; experiment (solid) on powder [6].

lowest is odd. For $S=1$, it is vice-versa. The $\Gamma_{5}$ states can be viewed as being of $p$-wave type.

For $S=0$, the lowest mode vanishes at the same $x$ as does the gap $\Delta$. So no additional instability occurs for $S=0$. This provides evidence against a competing singlet phase as presumed in Ref. [21]. There is, however, a salient instability for $S=1$ (Fig. 四) at $x=0.63$. This comes as a surprise since one expects in antiferromagnets binding effects to be largest for $S=0$. The singularity at $x=0.630(5)$ is very stable occuring in all non-defective Dlog-Padé approximants. We take the vanishing of a bound 2-triplet state with $S=1$ as evidence for strong attraction between triplets which are neither parallel $(S=2)$ nor antiparallel $(S=0)$. The attraction points towards a first order transition into a condensate of triplets occuring at much lower $x$ than thought previously [5]. The angle being neither zero nor $\pi$ corroborates a helical order if one adopts a classical view [22,5]. In the light of the instability for $S=1$ we interprete the findings by Koga and Kawakami 21] as indication of the same instability. It is so far not excluded that the bulk triplet condensate (the helical phase) is a singlet or that it can be linked to a singlet [23].

Next we determine $x$ and $J_{1}$ for $\mathrm{SrCu}_{2}\left(\mathrm{BO}_{3}\right)_{2}$. The steep decrease of the bound $S=1$ state enables us to fix $x$ very precisely. Based on ESR 20, FIR 24 as well as INS [19] we assume $\Delta=2.98 \mathrm{meV}$ and $\left.\omega\right|_{S=1}=$ $4.7 \mathrm{meV}$ leading to $x=0.603(3)$ and $J_{1}=6.16(10) \mathrm{meV}$. The 1-triplet dispersion is in excellent agreement with experiment (cf. inset in Fig. 3 and Ref. [11]). Raman scattering 15] provides further strong support because the energy of the $\Gamma_{3}$ singlet matches $30 \mathrm{~cm}^{-1}$ perfectly. The $\Gamma_{4}$ singlet at $25 \mathrm{~cm}^{-1}$ [25] is forbidden by symmetry 
since the Raman operator is effectively $\Gamma_{3}$.

In leading order $t / U$ the Raman operator $R=$ $\sum \gamma_{\mathbf{i}, \mathbf{j}} \mathbf{S}_{\mathbf{i}} \mathbf{S}_{\mathbf{j}}$ couples the same spins as the Hamiltonian. But only the antisymmetric $\left(\gamma_{1}=-\gamma_{2}\right)$ part of $R$ on the dashed bonds (cf. Fig. 1) creates excitations from the ground state. By geometry we have $\gamma_{1}^{\prime \prime}=\gamma_{2}$ and $\gamma_{2}^{\prime \prime}=\gamma_{1}$ so that the effective $R_{\text {eff }}$ is odd under R 1 and R2. But the projection of the vector potential $\mathbf{A}(\mathbf{A} \| \mathbf{E})$ on the bonds under study in the polarisation (ab) implies $\gamma_{1}=\gamma_{2}=-\gamma_{1}^{\prime}=-\gamma_{2}^{\prime}=\gamma \cos (2 \alpha)(\gamma$ microscopic constant, $\alpha$ angle in Fig. 1), i.e. an even component implying $R_{\text {eff }}=0$. On the contrary, polarisation ( $\left.\mathbf{a}^{\prime} \mathbf{b}^{\prime}\right)$ yields $\gamma_{1}=-\gamma_{2}=-\gamma_{1}^{\prime}=\gamma_{2}^{\prime}=\gamma \sin (2 \alpha)$ so that $R_{\text {eff }} \neq 0$. This finding agrees nicely with experiment where on $T \rightarrow 0$ the intensities almost vanish for (ab) but grow for $\left(\mathbf{a}^{\prime} \mathbf{b}^{\prime}\right)[15$. Additionally, $\gamma_{1}=-\gamma_{1}^{\prime}$ and $\gamma_{2}=-\gamma_{2}^{\prime}$ imply odd parity under SD so that $R_{\text {eff }}$ is indeed $\Gamma_{3}$, not $\Gamma_{4}$. Calculating the next $\Gamma_{3}, S=0$ bound state (less systematically) yields $45 \mathrm{~cm}^{-1}$ in good agreement with the experimental $46 \mathrm{~cm}^{-1}$ line, too.

We conclude that the 2D model (1) explains the lowlying excitations of $\mathrm{SrCu}_{2}\left(\mathrm{BO}_{3}\right)_{2}$ perfectly. Thermodynamic quantities like the susceptibility $\chi(T)$ require the inclusion of the interplain coupling $J_{\perp}$ which is fully frustrated not changing the dimer spins [26]. We have employed a Dlog-Padé approximant for the $1 / T$ expansion of the $2 \mathrm{D} \chi_{2 \mathrm{D}}$ 10 complemented by the condition $\Delta=2.98 \mathrm{meV}$. This ansatz works fine for $T>35 \mathrm{~K}$. The $3 \mathrm{D} \chi_{3 \mathrm{D}}$ is computed from $\chi_{2 \mathrm{D}}$ on the mean-field level as $\chi_{3 \mathrm{D}}^{-1}=\chi_{2 \mathrm{D}}^{-1}+4 J_{\perp}$. The inset in Fig. 1 shows that theory $\left(J_{\perp}=1.3(2) \mathrm{meV}\right)$ and experiment [6] agree without flaw above $40 \mathrm{~K}$. Our value for $J_{\perp}$ is significantly higher than the one in Ref. [26] due to different values of $x$ and $J_{1}$.

The above comprehensive analysis of bound states is a fine example of the efficiency of perturbation by unitary transformation [8] based on flow equations. This clear concept allows to distinguish uniquely sectors with different particle numbers and other different quantum numbers like the total spin. Here the concept was put to use to analyse the Shastry-Sutherland lattice as realized in $\mathrm{SrCu}_{2}\left(\mathrm{BO}_{3}\right)_{2}$. To our knowledge it is the first quantitative description of 2-particle bound states in 2D.

The symmetries of experimentally relevant states were determined. The reliability of the high order results allows to fix the experimental coupling constants very precisely $\left(J_{1}=6.16(10) \mathrm{mev}, J_{2} / J_{1}=0.603(3), J_{\perp}=\right.$ $1.3(2) \mathrm{meV}$ ). Thereby, different experiments (ESR, FIR, INS, Raman, $\chi(T))$ are explained consistently. We suggest to exploit the double degeneracy derived here to resolve different bound states at the border of the MBZ.

An unexpected instability for the $S=12$-triplet bound state is found at $x \approx 0.63$ indicating a transition to a triplet condensate probably related to the helical phase found previously [22,5]. We conjecture that this transition is first order occuring at lower $x$ than assumed so far. The vicinity of $\mathrm{SrCu}_{2}\left(\mathrm{BO}_{3}\right)_{2}$ to this transition suggests to attempt a closer experimental analysis. Pressure and/or substitution will certainly influence the ratio $J_{2} / J_{1}$. Thereby one may hope to scan through the transition and to examine the phase beyond.

The authors like to thank H. Kageyama and P. Lemmens for generous provision of data prior to publication and discussion. The work is supported by the DFG in SFB 341 and in SP 1073.

[1] C. K. Majumdar and D. K. Ghosh, J. Math. Phys. 10, 1388 and 1399 (1969); C. K. Majumdar, J. Phys.: Condens. Matter 3, 911 (1969).

[2] B. S. Shastry and B. Sutherland, Physica 108B, 1069 (1981).

[3] P. M. van den Broek, Phys. Lett. 77A, 261 (1980).

[4] B. S. Shastry and B. Sutherland, Phys. Rev. Lett. 47, 964 (1981).

[5] E. Müller-Hartmann, R. R. P. Singh, C. Knetter, and G. S. Uhrig, Phys. Rev. Lett. 84, 1808 (2000).

[6] H. Kageyama et al., Phys. Rev. Lett. 82, 3168 (1999).

[7] S. Miyahara and K. Ueda, Phys. Rev. Lett. 82, 3701 (1999).

[8] C. Knetter and G. S. Uhrig, Eur. Phys. J. B 13, 209 (2000).

[9] F. Wegner, Ann. Phys. (Leipzig) 3, 77 (1994).

[10] Z. Weihong, C. J. Hamer, and J. Oitmaa, Phys. Rev. B 60, 6608 (1999).

[11] C. Knetter and G. S. Uhrig, in preparation.

[12] In the language of the unitary transformation a virtual process is given by a monomial $T(\mathbf{m})$ [8].

[13] S. Miyahara and K. Ueda, Phys. Rev. B 61, 3417 (2000).

[14] T. Momoi and K. Totsuka, Phys. Rev. B 61, 3231 (2000).

[15] P. Lemmens et al., cond-mat/0003094.

[16] Y. Fukumoto, cond-mat/000411; Eq. (10) is confirmed. But we have deviations in most of the $5^{\text {th }}$ order terms in Table I.

[17] Explicit results will be made available electronically under "www.thp.uni-koeln.de/ gu" or "www.thp.unikoeln.de/ ck".

[18] Phase Transitions and Critical Phenomena, edited by C. Domb and J. L. Lebowitz (Academic Press, New York, 1989), Vol. 13.

[19] H. Kageyama et al., submitted to Phys. Rev. Lett.

[20] H. Nojiri et al., J. Phys. Soc. Jpn. 68, 2907 (1999).

[21] A. Koga and N. Kawakami, cond-mat/0003435.

[22] M. Albrecht and F. Mila, Europhys. Lett. 34, 145 (1996).

[23] On large systems even the Néel state can be approximated by $S=0$ states, cf. S. Liang, B. Douçot, and P. W. Anderson, Phys. Rev. Lett. 61, 365 (1988).

[24] T. Rõõm et al., cond-mat/9909284.

[25] The small shoulder at $25 \mathrm{~cm}^{-1}$ may be a trace of the $\Gamma_{4}$ singlet due to higher order processes or a singlet-triplet excitation.

[26] S. Miyahara and K. Ueda, cond-mat/0004260. 